\title{
Multimedia augmented reality game for learning math
}

\section{Cristina Rebollo $^{1} \cdot$ Inmaculada Remolar $^{1}$ (D) $\cdot$ Veronica Rossano $^{2} \cdot$ Rosa Lanzilotti $^{2}$}

Received: 31 July 2020 / Revised: 10 February 2021 / Accepted: 10 March 2021 /

Published online: 31 March 2021

(C) The Author(s), under exclusive licence to Springer Science+Business Media, LLC, part of Springer Nature 2021

\begin{abstract}
The traditional method for learning the multiplication tables is a repetitive and boring task. Teachers try to find new methods to motivate children in this tedious duty, and one of the lines to consider is to integrate en- tertainment into educational processes. This work presents a new multimedia interaction approach in order to allow children to practice these math opera- tions and have fun. The learning process has been gamified by means of two mini-games designed for mobile platforms, based on meromictic or repetitive learning. The genre of these mini-games have been selected according to children preferences: one turn-based fighting and other throwing-objects game. A series of proposed multiplications have to be solved during the play to per- form the player actions. Moreover, in order to support learning engagement, both have been visualized through Augmented Reality, combining real and virtual reality. This paper discusses the good results of mixing entertainment with some learning tasks, due to the engagement of the children to the mobile based games. A pilot study has been performed in order to evaluate the learn- ing effectiveness and usability of the proposal. Results support that playing the video games makes this tedious multiplication practice more enjoyable and attractive for children so they improve their math skills.
\end{abstract}

Keywords Serious games · Engagement $\cdot$ Education $\cdot$ Augmented reality

Inmaculada Remolar

remolar@uji.es

Cristina Rebollo

rebollo@uji.es

Veronica Rossano

veronica.rossano@uniba.it

Rosa Lanzilotti

rosa.lanzilotti@uniba.it

1 Institute of New Imaging Technologies, Universitat Jaume I, 12006 Castellón, Spain

2 Department of Computer Science, University of Bari A. Moro, 70125 Bari, Italy 


\section{Introduction}

Learning motivation is a topic of concern among teachers so different ways of improving it has been studied in the literature $[15,16]$. Some works, such as those presented in [21, 24], demonstrate that using games improves the level of motivation and commitment of the students, since they can combine en- tertainment with their own training activities. Digital devices, and especially mobile ones, are very popular with young people and most of the recent re- search have used them as platforms for game-based learning [24]. According to [30], mobile learning includes the concept of anytime/anywhere [12], and allows leisure moments to be used as an opportunity for learning. In addition, mobile or wireless devices are currently available to almost everyone, because of the great popularity of the smartphones in the society. This fact avoids increasing the costs of the experiments if they are used in any research.

Augmented Reality (AR) combines real and virtual objects with a three- dimensional registry, making it possible in some cases to interact in real time [2]. This technique is presented itself as a good tool to increase engagement to the use of the applications developed [3, 32]. Moreover, AR converts learn- ing situations into contextual situations, which usually improves educational praxis [9]. The concept of "presence" in these games makes that users feel that the game is being developed in the place they are physically located, so the sense of 'being there' in both real and virtual worlds while playing the game has been shown to improve the learning environment [3, 32]. Video games that include $\mathrm{AR}$ in the field of education have reached areas as different such as History, English, Environmental Sciences, and of course, the one related with STEM (Science, Technology, Engineering and Mathematics) [1, 4].

Within the field of mathematics, a highly repetitive and highly practical task is learning the multiplication tables. Traditionally, it consists of repeating each of one of the multiplications infinitely, first by memorizing them and then by carrying out a multitude of multiplications on a paper sheet, so the children who are in that process usually find it quite boring. More enjoyable and fun ways to memorize the different tables have been found, such as reciting them as a song, or using "Memory" games consisting of matching the multiplication and its result. However, the fact that the current generation has grown as digital natives has made the option of consolidating their knowledge in this field through the use of video games an option to consider.

This paper proposes an application that gamifies the practice of the multi- plication tables aimed at children in Primary Education, making this task less monotonous. The main target of the work is to practice the multiplication op- erations that they have learnt at school, so they can consolidate the acquired skills. Moreover, the mobile application has been designed trying to promote the interest of the final users. For this purpose, design principles for children's learning games have been used in this work [23], following the indications of a Human Centered Design (ISO 9241-210: 2019) [8].

As a result of this analysis, a video game has been designed with two mini- games. The first, Battle against the Colossus is a turn-based fighting game between two opponents. The second, Save the Planet! is a throwing-object game that presents the idea of saving the Earth to continue supporting life. Also the mini-games offer the possibility to the user of selecting the avatar that represents the player, a male or female character. After the game play ends, a report of successes and failures per game are displayed.

These mini-games are visualized in AR, so children can see the operations to solve and the game to play combined with the same real environment they are located in. Using this technology has demonstrated that it increases the engagement of the applications [10]. 
Different qualitative and quantitative formative studies have been planned to evaluate the effectiveness of learning and the usability of the different proto- types. Obtained results support that using this technology has positive impact on the acquisition of multiplication tables by the children.

The rest of the work is organized as follows. Section 2 introduces some ed- ucational experiences visualized using AR. Section 3 analyzes the preliminary study of the application. Section 4 introduces it, analyzing its game architec- ture and the scene game visualization. Section 5 details the generation and management of the multiplication operations. Then, Section 6 describes the mini-games Battle against the Colossus and Save the Planet!. Section 7 an- alyzes the experiments performed to analyze the developed application and the results obtained are discussed in Section 8. Finally, conclusions and future work are presented in Section 9.

\section{State of the art}

The use of AR complements traditional teaching methods and materials with some virtual content. The fact that only a smartphone is required to visualize digital objects over real content makes this technology appropriate to be used in education with the aim of improving the learning and motivation of the students [26]. Some popular applications have appeared to learn languages such as TeachAR or HELLO [17].

In the context of mathematics, AR has been widely applied to learning geometry. Geo + [22] is addressed to reinforce the learning of the main solid figures on a mobile device for 3rd grade Primary students. Results presented by the authors confirmed that children were attracted to the application and had fun, although the evaluation method was not convincing, since the results of both the pre-test and the post-test were very high in both cases. ARGeo [11] is an application for high school students used to practice the basic principles of geometry, comparing the effectiveness of learning the AR-based activity with the Web-based activity. It concluded that users could manage significantly better with the AR app, and higher levels of motivation were promoted, in terms of attention, relevance, condescension and satisfaction. In order to visu- alize, manipulate and explore concepts such as linear, quadratic, exponential and trigonometric functions (sine and cosine), Foothmath [5] was created for secondary school students. A soccer game represented in $3 \mathrm{D}$, that deals with manipulating different functions and exploring them using parameters with different values to score goals. An exploratory study was carried out with a quantitative and qualitative methodological approach to this game [6], con- sidering FootMath a promising and innovative tool to be incorporated into real settings for teaching mathematics.

The aforementioned applications work with AR and markers, but the trend in recent years has been to replace markers with surface recognition. Using this technique, GeoGebra AR [27] allows students to mathematically construct $3 \mathrm{D}$ models virtually placing them in the real world and virtually testing the ac- curacy of the created models, allowing to improve them. ARMaths [14] allows us to work with concepts such as addition, subtraction, multiplication, etc. The manipulation of tangible and virtual objects in a mixed reality and in a real environment is compared in the work. Results indicate that the inter- action with a touch screen promotes collaboration and reflection by stopping impulsiveness.

The serious game presented here has been developed following the design principles of learning games with AR for mobile aimed at children [13]. In addition, taking into account the 
efficiency of AR in the field of education, this video game promotes the motivation of learning of multiplication tables using the latest technique in AR: surface recognition.

\section{Preliminary study}

As it has been previously said, the mobile application has been designed fol- lowing the indications of a Human Centered Design (ISO 9241-210: 2019) [8], so different meetings and studies have been carried out to adapt the design to the characteristics of the final users.

On one hand, the genre of the mini-games had to be determined. The idea of the developed application was that children do not feel that they were studying while they were using the mobile application, so a survey was designed targeting parents and teachers, in order to know the genre of the most popular computer games that children ranging in age from 8 to 10 years old use to play. All data were collected using Google Forms. The five genres selected by parents and teachers were as follows:

- Platform Games

- Throwing-object Games

- Racing Games

- $\quad$ Turn-based Fighting Games

- Puzzle Games

A group of 50 children in that age range answered a new survey with these selected genres, being 25 boys and 25 girls. They all rated their preferences of the games following a Likert scale. Results demonstrate that they prefer the genres of Throwing-object (30\%) and Turnbased Fighting Games (35\%). The games presented in this work were designed following the game mechanics of both selected genres.

On the other hand, another study was developed to design the educational part of the work. Some professionals in education participated giving their opinions and experience about the time that they considered was the most appropriate for children to answer the multiplications. This group was com- posed by 15 primary school teachers, with more than 10 years of experience. This topic was discussed with all of them and finally it was established that 10 seconds was the maximum period of time that children require to think about an answer without losing focus on the question. Also, another discussion was about the numbers offered as options to be the correct answer of a determined multiplication. They had to be the result of other multiplications. Then, those numbers seem familiar to them as possible answers.

\section{The developed AR application}

Two mini-games are offered in the mobile application, Battle against the Colos- sus, a turnbased fighting game, and Save the planet!, an object-throwing game. In both, the player visualizes the game scene over the real environment she/he is located, reproducing the game on a flat area. The art used in both games has been obtained from [19, 25, 28] always choosing models composed by a low number of polygons. The animations have been done with Mixamo [18], and the text has been generated with the CoolText tool [7]. 
The mobile application starts with a general presentation. Next, the pos- sibility of selecting one of the two mini-games is offered (Fig. 1 left) and, after this, the user has to choose the character he/she wants to play with, a hero or a heroine (Fig. 1 right). Then, a surface has to be detected by the mobile device to visualize the mini-game.

The interaction of the user is related with the operations of multiplying from this point of the application. Depending on the success in solving them, some actions will be performed in the mini-games.

Both mini-games share the general architecture and the educational part of the games. The only difference between them are focused on the game play.

\subsection{The game architecture}

The application has been created for mobiles with Android and iOS operat- ing systems, developed with UNITY 2019.2.3 version and managing the RA with Vuforia plug-in that includes the surface detection Ground Plane, and camera management [31]. The architecture of this application is graphically represented in Fig. 2.

GameMultiplyAR is the main component of the application, composed by the Hierarchy and the Components elements. All the graphic elements and the GameObject are located in the Hierarchy to be able to configure the scene in the application. The Components contain all the necessary scripts to imple- ment the different functionalities of the application. UnityLibrary is the set of all the components of the Unity game engine that are used to create the GameObject and the scripts. Vuforia is the Unity plugin used to build and manage Augmented Reality contents.

\subsection{Scene game visualization}

Some works in the literature demonstrate that Augmented Reality visualiza- tion increases the engagement of the users to the application $[3,32]$. That is the target of the developed work: children spending time using it without getting bored.

To perform this visualization, it is necessary firstly to detect the surface in the real environment that makes it possible for objects to appear over it. The Ground Plane utility supports detection and tracking of horizontal surfaces, using the Vuforia API Smart Terrain together with tracking devices. It allows viewing AR without the need of traditional markers: it is only required to use the camera of the device to detect surfaces to place the scene on them. The utility is capable of detecting in real time a flat surface by means of the images obtained by the camera, and then positioning the digital content anchored to that surface, so the user has only to locate with the device's camera a flat surface of around $1 \mathrm{~m}^{2}$ that is correctly illuminated in order to allow instantiating the scene on it. This
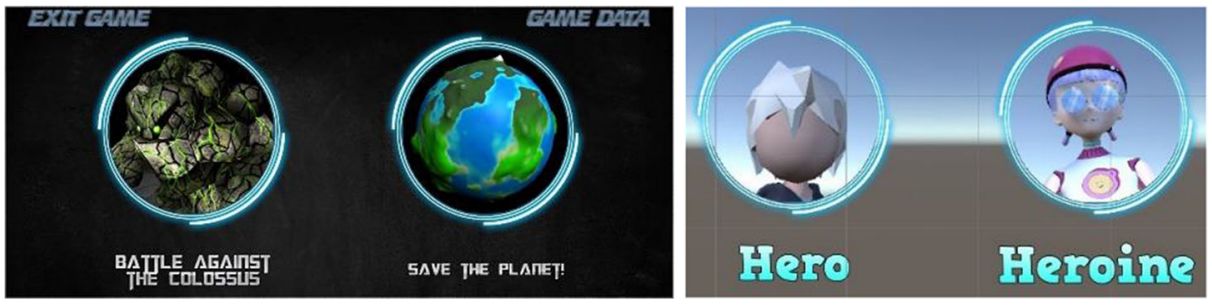

Fig. 1 Selection screen for the mini-game (left) and the character (right) 


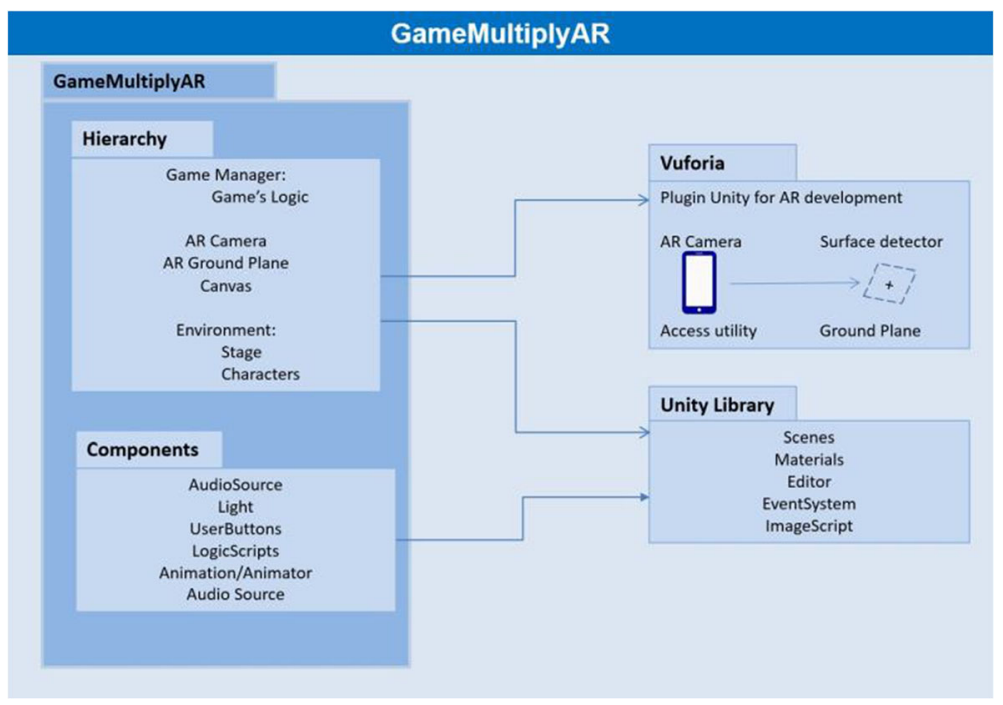

Fig. 2 AR application architecture

technique focuses on recognizing and interacting with the environment, keeping the programmer out of the real world detection process.

\section{Management of the multiplication operations}

The multiplication operations are automatically generated. The pseudo code of the process is shown in Algorithm 1. In order to avoid practice many times with the same table, a method has been proposed to control their repetitions, limiting them to a maximum of 3 times per minigame. So, the maximum number of multiplications to solve are 30 (Multiplications solved).The following variables are defined to better explain the process:

- Used Table: A 10-dimensional array that stores the number of times each table has appeared.

- Performed Operations: A $10 \times 10$ dimensional matrix that collects the pos- sible operations between the tables. In this one, the ones that come out are marked.

To propose a multiplication, the function (Generate number()) generates the first number that represents the table that is going to be practiced. This number is then checked to know if it has already been used 3 times, so that table number is blocked. If this has been the case, another number will be generated again. If this number is available, it will be increased by one the times that the table has been used (Used Table) and the number of performed multiplications.

To generate the second number, by which the table is multiplied, the matrix of available operations is checked (Performed Operations), in which opera- tions are marked as they come out. Function Unused Num randomly chooses a number from those that remain unused within the row of the matrix of the current table. Then, the operation that involves the two selected numbers is marked in the matrix as performed by Function Mark Operation. This pro- cess, in addition, guarantees that there are no repeated operations in the same mini-game. Once the two 
values are obtained, they are offered to the user, appearing in their corresponding area on the multiplication zone of the game play.

The application also has a vector that stores the results of all the possible multiplications. The numbers shown in the application as possible results are extracted from this vector, among which is the correct result. This process ensures that the numbers that appear as possible results are in fact results of other multiplications, not impossible numbers that are easy to discard by the users. The application will randomly order the four values, knowing the position of the correct value.

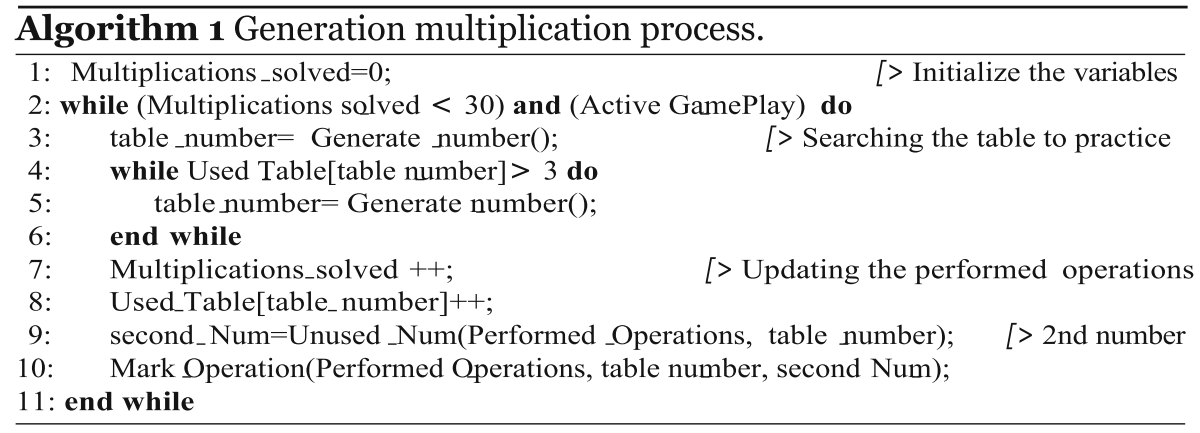

Each time a certain operation is answered and it is analyzed whether or not it is correct, the time bar assigned to the solving time is initialized and a new multiplication operation is generated and available for being solved again by the user. Every time the user selects a result, or when the response time ends, the solution is visually updated in the multiplication operation area in the game play (Fig. 3):

- If the number pointed out is the right result, the selected box turns green.

- If the user has failed, the wrongly selected box containing the number turns red and green the correct answer so that the child knows the correct result of the operation.

Then, the actions of the game associated with the success or the failure of the answer, such as animations and sound effects, are triggered. Once the mini- game has finished, different possibilities are available: play again to one of the mini-games or to quit the application. In the first case, a graphic evaluation of the results are shown (Fig. 4 up). All the possible multiplications are shown in an image where the ones that the user has hit are colored in green and the ones that have failed in red. This information is also summarized as numerical data.

If the child decides to quit the application, it is offered a graphical analysis comparing the results of the first and the last game played (Fig. 4 down). Both tables include the total number of failures and hits in both situations, so the child can appreciate the evolution in the trainee-ship.

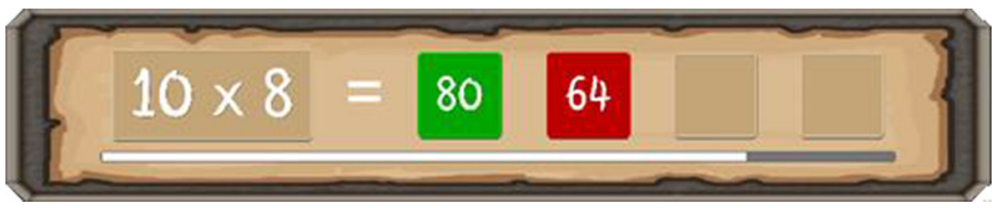

Fig. 3 Graphic result of an operation when a wrong result has been pointed out 


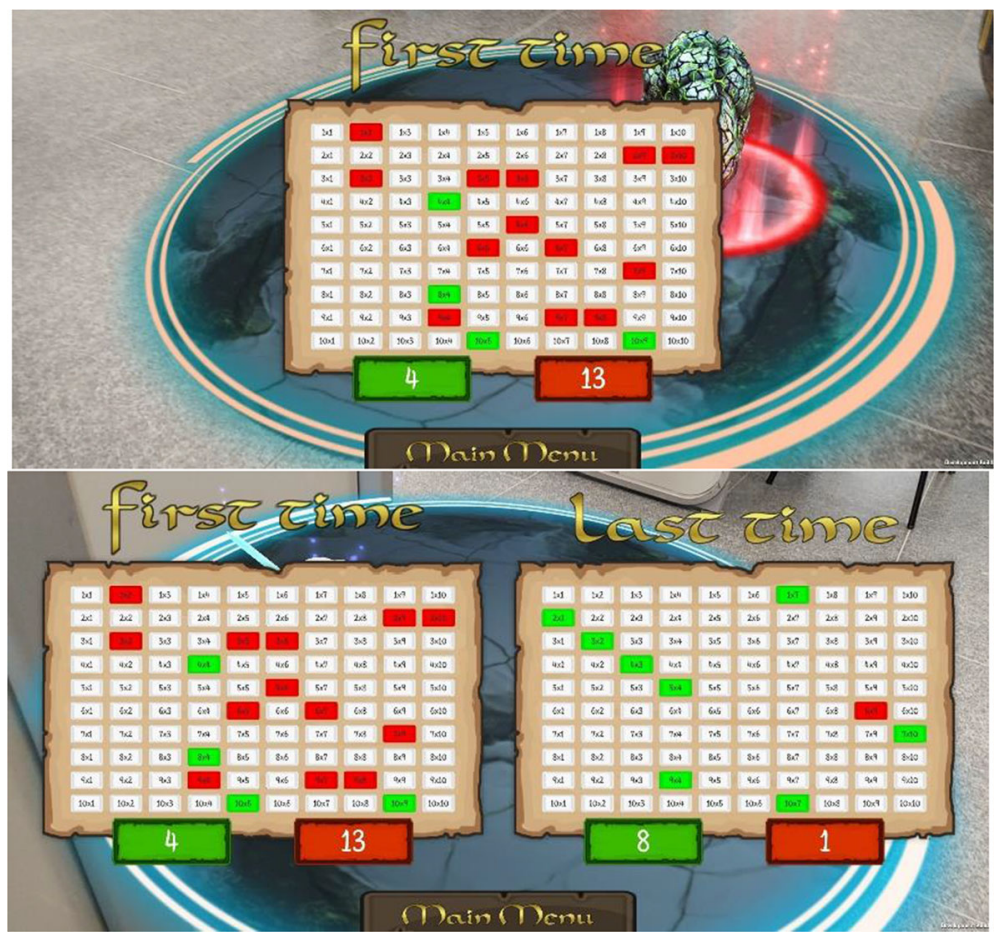

Fig. 4 Partial graphic results when finishing a mini-game (top) and the final summary of the application (down)

\section{The designed mini-games}

Two mini-games have been designed according to the genre preferences of the children: turnbased fighting and throwing-object games.

\subsection{Battle against the Colussus game}

Battle against the Colussus is a turn-based fighting game that involves the character selected to represent the player and the Colossus, the adversary. The game play includes the fighting area and the multiplication to be solved, located at the bottom of the screen (Fig. 5). The objective of this game is to end the life of Colossus.

The game consists of two alternating turns: in one turn the player attacks and in the other one, he/she tries to avoid the attack of the Colossus. A multiplication operation has to be resolved each turn. The only action to take in the game by the user is to find the result of the proposed multiplication. Four numbers are offered as possible solutions, only one of them being correct. It is also considered an error not to provide an answer in 10 seconds, the period of time advised by the educator experts. A time bar at the bottom of the screen reflects the elapsed time.

Different actions will be triggered in the game depending on whether the user guess correctly or incorrectly the result and, also, depending on whether the player is attacking or defending him/herself: 


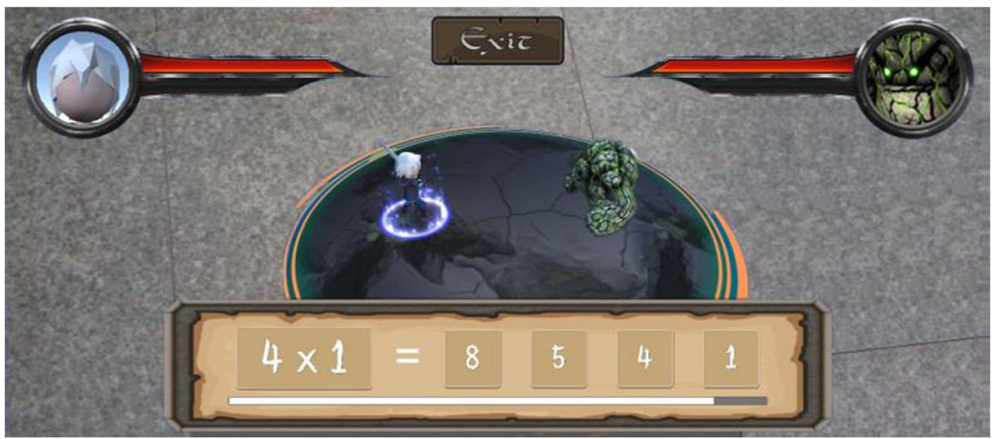

Fig. 5 Game play scene of Battle against the Colossus

- If the player is attacking: If the user points out the right result of the pro- posed multiplication, the player will beat the enemy, reducing his lifetime. Otherwise, the attack will fail and the Colossus will avoid the life reduction.

- In the defending turn: if the number selected by the child is correct, the player will stop the opponent's attack. Failing the result of the operation will leave the character player defenseless so its life will be reduced.

The mini-game ends if one of the two combatants run out of life, an option reflected in their life bar, or when 30 multiplications are answered without either being defeated, being considered a draw.

\subsection{Save the planet! Game}

This mini-game is an object throwing game whose objective is to prevent the planet from destroying itself due to the contamination by residues. Users can save it by solving the proposed multiplications.

The game play scene (Fig. 6) includes some figures that represent the player, the waste container, the planet and its life bar, 30 cans at the top of the screen and the area

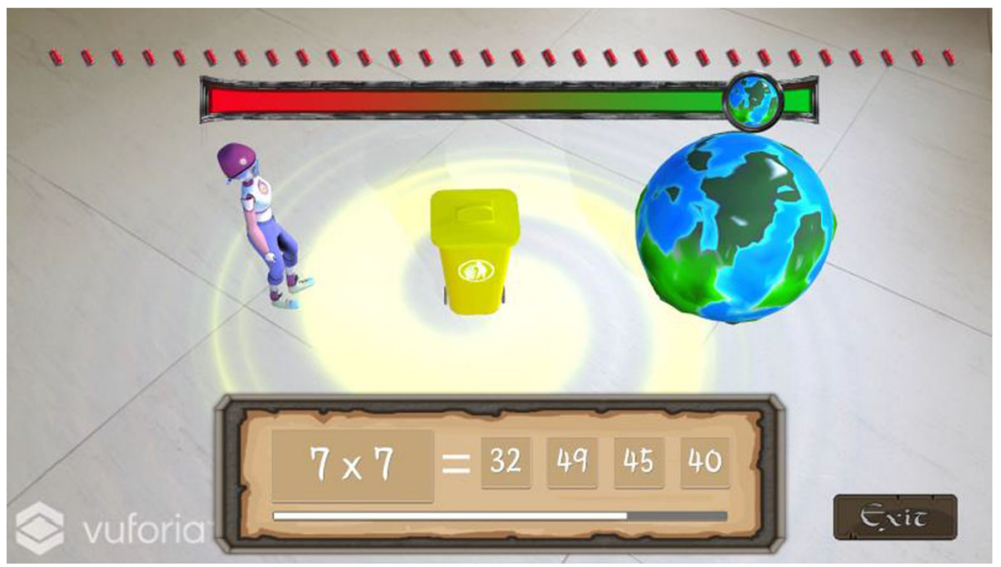

Fig. 6 Scene from the "Save the planet!" game 
with the multiplication to be solved. As in the previously analyzed mini-game, the child has to choose a number as the right result of the multiplication. Different actions will be triggered in the game depending on whether user hit or miss it the multiplication:

- If the number pointed out as a result is correct, the player throws the can into the recycling bin. The planet recovers life with this action until it becomes completely healthy.

- If the user fails, the player throws the can at the planet. The contamination by residues increases and the planet is damaged, being able to explode.

Once the user selects a possible solution or the response time finishes, two main actions are performed: the result is updated by changing the color ac- cording to the success or the failure in the selected number and, moreover, one of the cans located in the upper area of the screen is eliminated. The anima- tions that cause the player to throw the can are performed. Green particles will be shown if the can has got into the container, or red if it has collided with the planet. In both cases, the thrown can is destroyed after the collision. Also, the visual appearance of the planet is updated depending on its life.

The life bar indicates the situation of the planet as it is played. Initially, the life marker is located in the middle, and will move to the right or the left depending on the successes or failures in the multiplications. It must be considered that if the user fails the result, the planet loses more life than it gains if he/she hits. The game ends if the planet explodes, if it reaches $100 \%$ life, or when 30 multiplications have been answered without the planet exploding.

Save the planet! also has two relevant technical aspects. On one hand, the scene visualization process has been accelerated by using Shader Graph [29], that allows to generate the spatial vortex effect that acts as the "base" of the scene and makes it possible to color the planet. On the other hand, the creation and modification of the planet is carried out using procedurally generation techniques [20].

Modeling the planet by code makes it possible to modify it in real time. Four states have been determined for the planet, according to the associated life: healthy, desert, burned and, finally destroyed by an explosion (Fig. 7). In a pre-process, the parameters used to generate these four states are calcu- lated and stored. The life of the planet is taken into account every time the player throws a can, to get the new shape and color of the representation. The programmed code slowly interpolates the previous and current state, avoiding popping effects.
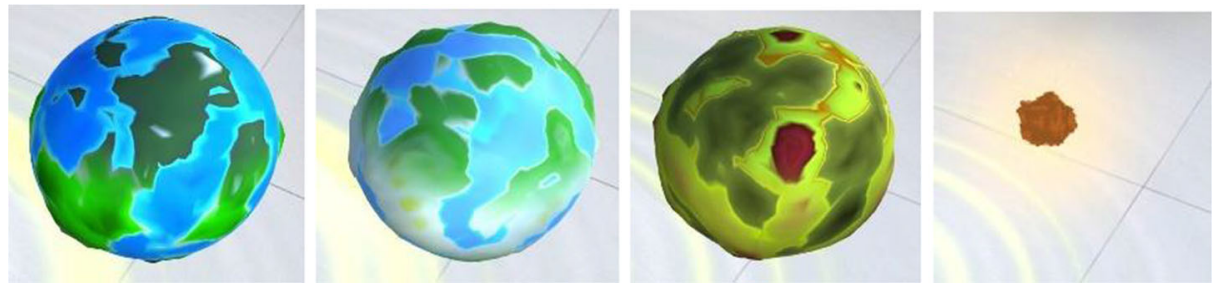

Fig. 7 Phases of the planet: healthy, desert, burned and destroyed 


\section{Pilot study}

The Covid-19 pandemic has prevented all the planned evaluation steps. How- ever, an evaluation study was carried out on the first version of the mobile app. Two working hypotheses have been defined:

- H1: The presented digital practicing method positively impacts on the acquisition of multiplication tables.

- $\mathrm{H} 2$ : the AR visualization promotes the practicing engagement more than the traditional method.

\subsection{Participants and design}

The study involved two groups of children ranging in age from 8 to 9 years old. Previously, their parents were informed about the aim and the method of the study, and they gave their consent for their sons and daughters to take part in this study. All of them had already acquired the multiplication tables in previous traditional learning activities at schools. As it was previously said, the target of the application has been to practice out of the school and consolidate their knowledge.

A between-subject design was applied, with the learning as an independent variable. Two groups were formed. These are detailed in Table 1. Specifically, the experimental group (EG), composed of 18 children (11 boys, 7 girls), was in charge of testing the AR application. They used the application at home, using their parents' mobile in some cases or some available tablets in others. The control group (CG) was composed of 19 pupils (7 boys, 12 girls); children in this group practiced the math operations using the traditional method, they were given a notebook with some operations to solve.

\subsection{Data collection}

Two tests were designed: the pre-test to be submitted before the experiment, and the post-test after it. They are detailed in Tables 2 and 3. In order to measure the learning effectiveness of the application, the questions to be filled in by the participants were collected using paper sheets.

The pre-test designed for the CG was composed of 14 questions: 3 deal- ing with demographic data, 1 question related to self-assessing knowledge of multiplication tables in general and the last one related to the self-assessing knowledge per every one of the ten multiplication tables. Finally, to check the knowledge of the multiplication tables before the experiment, every child performed 10 exercises. The hits and the failures were stored in this pre-test.

Table 1 Distribution of the participants in the experiment

\begin{tabular}{lll}
\hline Groups & Practice method & Sample \\
\hline Control Group (CG) & traditional method & 19 pupils (7 boys, 12 girls) \\
Experimental Group (EG) & developed application & 18 children (11 boys, 7 girls) \\
\hline
\end{tabular}


Table 2 The designed pre-test

\begin{tabular}{lll}
\hline Group & Pre-test & Related Subjects \\
\hline CG & 14 questions & 3 dealing with demographic data \\
& 1 related to self-assessing of the current knowledge of the tables \\
& 10 related to the self-assessing of the current knowledge of every table \\
& 10 random multiplication operations \\
& 3 dealing with demographic data \\
EG & 15 related to self-assessing of the current knowledge of the tables \\
& & 10 related to the self-assessing of the current knowledge of every table \\
& 1 related to the self-assessing technological abilities \\
& 10 random multiplication operations \\
\hline
\end{tabular}

The pre-test designed for the EG was the same, but we added some ques- tions about selfassessment of the user's technological abilities, taking into account computers, tablets or smart-phones. This was important to analyze usability data.

The other designed questionnaire was prepared to be filled in once the ex- periment has finished. In the post-test the main question was posed to measure the learning effectiveness, related to the current self-assess of the knowledge acquired during the exercise (using digital or traditional method). Pupils in the experimental group underwent a usability test (13 questions) in order to assess the users' appreciation of the game and answered some questions related to the engagement ( 2 questions) due to the AR.

Finally, in order to evaluate the acquired knowledge, both groups solved 10 exercises to compare the results obtained with the previous test.

\subsection{Procedure}

Initially, all participants underwent the pre-test. The tests were anonymous; a code was assigned to each participant to allow pre-test and post-test data to be then correctly compared. Regarding the children that were going to practice with the application, it was asked to children's parents to download and install the game on their own devices. Participants in both groups had to practice the multiplication operations for half-an-hour a day for one week. The participants of the EG interacted with the game, while the participants of the CG solve the multiplications in a notebook administered by their parents. This time was controlled by the parents for the participants of both groups.

Some data had to be stored during the experiment. Mainly, the number of times the child has practiced every multiplication table. These data were collected in order to normalize data, since if a user exercises a few times with a specific table, it will be highly likely s/he's going to

Table 3 The designed post-test

\begin{tabular}{lll}
\hline Group & Post-test & Related Subject \\
\hline CG & 1 question & 1 related to the self-assess of the acquired knowledge due to the test \\
& 10 exercises & 10 random multiplication operations \\
EG & 16 questions & 1 related to the self-assess of the acquired knowledge due to the test \\
& 10 exercises & application 13 questions related to the Usability test \\
& 10 random multiplication operations \\
\hline
\end{tabular}


Table 4 Data retrieved in the test

\begin{tabular}{lll}
\hline Group & Data collected & Collection method \\
\hline CG & Number of times each table has been used & Parents \\
EG & Number of times each table has been used & Application \\
& Number of times every mini-game has been played & Application \\
\hline
\end{tabular}

get that multiplication table wrong. As it is shown in Table 4, the parents collected these data in the CG group, while the application was prepared to store these numbers in the EG group, so they were quickly collected. Regarding the experimental group, it was also retrieved how many times the user had played every mini-game.

At the end of the week the post-test was administered, and all the data were gathered.

\section{Results and discussion}

\subsection{Learning effectiveness}

The mean age of the participants was 8.5 years old, for both groups. The self- assessment of multiplication table knowledge was measured for all pupils. This revealed that pupils were aware of the fact that they needed to practice, and that they were comfortable with some multiplication table (i.e. 1, 2, 5, 10), and less with others (in particular, 6, 7, 8, 9), as shown in Fig. 8.

As previously pointed, in the EG some questions in the pretest were posed about pupils' familiarity with technologies. All of them use the smartphone, $61 \%$ of the sample use also the tablet and $67 \%$ of pupils also use the pc, only 7 children use all three devices. In order to evaluate their ability in using the technologies, in the pre-test participants were asked to selfassess (using a score from 1 to 5) their ability in using each single device. As expected, pupils were very familiar with tablets (3.7 out 5) and smartphones (3.9 out 5) since they are the most used devices in this age range (Fig. 9). Only 7 out of the 18 pupils had previously used an augmented reality application.

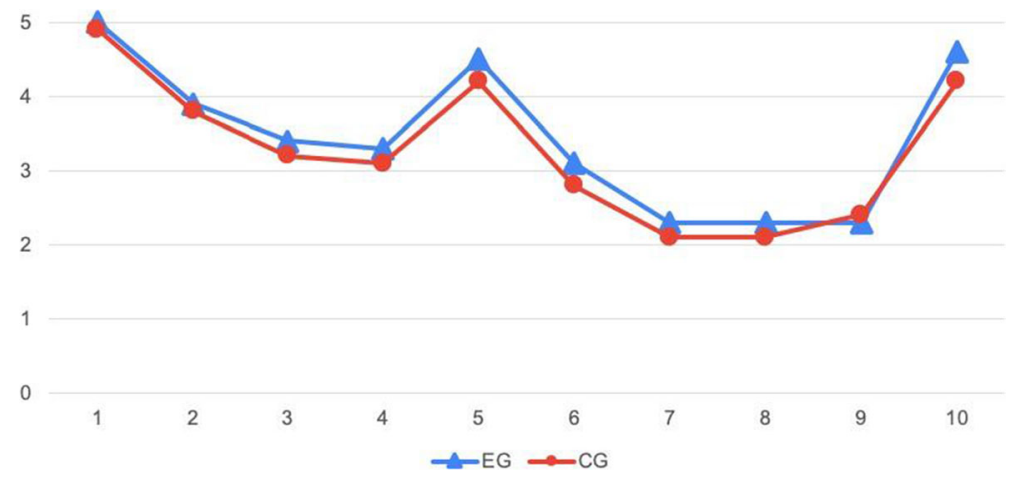

Fig. 8 Knowledge of multiplication tables: the abscissa axis represents the table and the ordinate axis the selfassessment related to it 


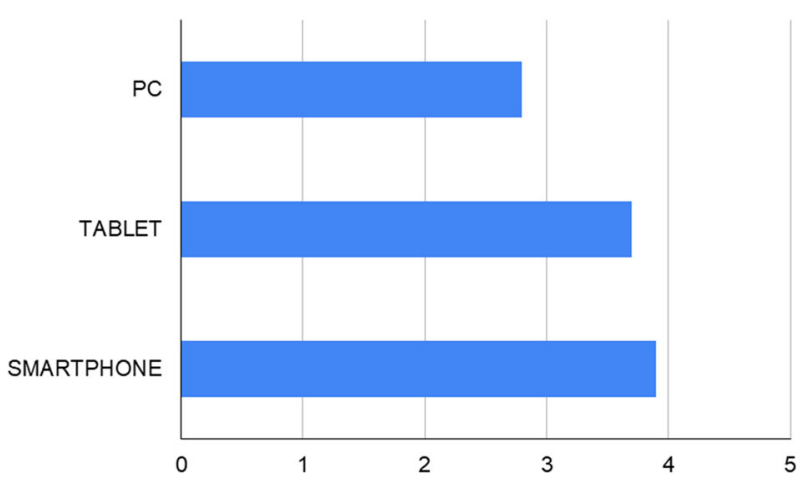

Fig. 9 Pre-test self-assessment of users' technological skill using a Likert scale (0: never used; 5: frequently used)

Participants were asked to self-assess their knowledge on the multiplica- tion table before the use of the application in the case of the EG or solving the operations in the notebook in the case of the CG and at the end of the study. Participants had to assess their knowledge with a 5point scale $(1=$ no knowledge, $5=$ good knowledge $)$. Participants of both groups perceived an improvement of their knowledge on the multiplication tables. In particular, the score of the knowledge of the EG participants before the use of the application was on average 3.3 ( $\mathrm{SD}=$ 0.77 ) while after the use of the application slightly increased to 3.83 ( $\mathrm{SD}=0.7$ ). The participants of the CG perceived a greater improvement than the participants of the EG starting from an average score equals to $2.95(\mathrm{SD}=0.7)$ and reaching a final average score of 3.53 (SD $=1.02$ ). This result highlighted that pupils in EG did not actually perceive the interaction with the game as a learning activity so much that they did not think they had improved their knowledge on the multiplication tables.

Learning effectiveness was assessed through two repeated tests. The tests included 10 exercises addressing the 10 multiplication tables. The rationale for the repeated-measure test was to analyze the effectiveness, measured in terms of learning gain, of the method (i.e. digital or traditional) used for consoli- dating the knowledge acquired during the school. The learning gain score was computed, subtracting the number of correct answers received from the posttest to the number of the correct ones got from the pre-test (Fig. 10). The two learning gain scores were subjected to an independent t-test with learn- ing method (digital- vs. traditional) as between-subjects factor. The analysis revealed no significant effect for method, $\mathrm{t}(35)=-$

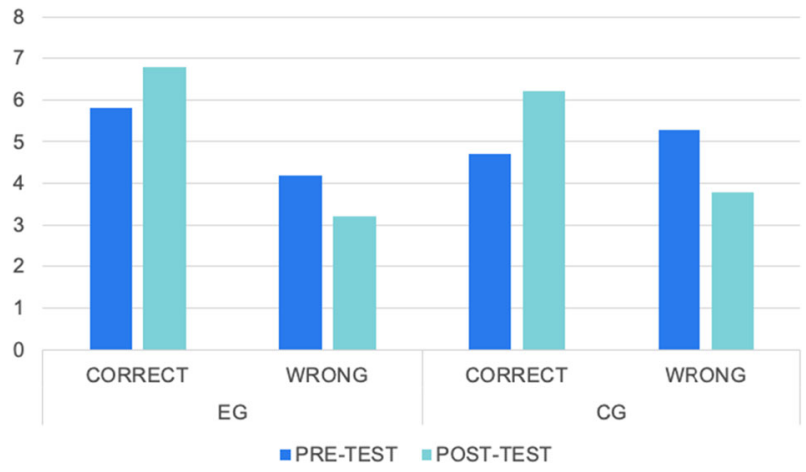

Fig. 10 Number of correct and wrong answers to exercises in pre-test and post-test 
$0.99, \mathrm{p}=.33$, despite dig- ital method $(\mathrm{M}=1.05, \mathrm{SD}=1.16)$ attaining higher scores than traditional method $(\mathrm{M}=1.42, \mathrm{SD}=1.07)$. The experimental data confirm the first hy- pothesis (H1), i.e. the digital method positively impacts on the acquisition of multiplication tables as well as the traditional method. This result is very positive, meaning that the use of technologies does not distract learners from their final goal. Distraction indeed is one of the main drawbacks that people against the use of technologies in learning activity uphold. Learning activity supported by the use of computers in general, and serious games in particular, as effective as the traditional ones.

One of the critical points of the game, could be due to the randomly choice of the multiplication proposed. The game could propose several times the same table giving the opportunity to practice that table more than others. For this reason, the game records how often a table is used in the game, in this pilot study the average number that each table is proposed has been calculated, and for all the tables the mean goes from 24 to 28 times. On average each child has solved 270 multiplication.

Moreover, to answer to the second experimental hypothesis (H2: the digital method promotes the engagement more than the traditional one) in the post- test was administered the question "Would you have liked to spend more time in doing this activity?", positive answers were the $67 \%$ in the EG sample vs the $21 \%$ in the CG sample. A chi-square test showed a significance statistics difference between the engagement felt by the participants in the two different groups $(\mathrm{X} 2=0.0081, \mathrm{p}<.05)$. In other words, the training multiplication table with the game resulted to be more engaging and enjoyable than the traditional refresher at home. The results were confirmed by the explicit comments from the children.

\subsection{Usability}

The usability test was composed of 13 questions, using a 5-point Likert scale (from strongly agree to strongly disagree), aimed at discovering if the partici- pants appreciated the game, and which game between the Battle against the Colossus and Save the planet! (Fig. 11 left). Data show that females pre- ferred the game Battle against the Colossus, whereas males preferred Save the planet!. The questionnaire was composed of 5 questions addressed to measure the comfort of the device and of the gaming approach to practice multiplication tables, and 8 questions aimed at measuring the usability of the specific game chosen. As it was easily predictable, the majority of users had no difficulty in using the smartphone (Fig. 11 right), 94\% of the sample was not distracted by phone use. The $94 \%$ of the sample declared that it was not complicated at all to calculate the result of the multiplication tables in a short time. This result
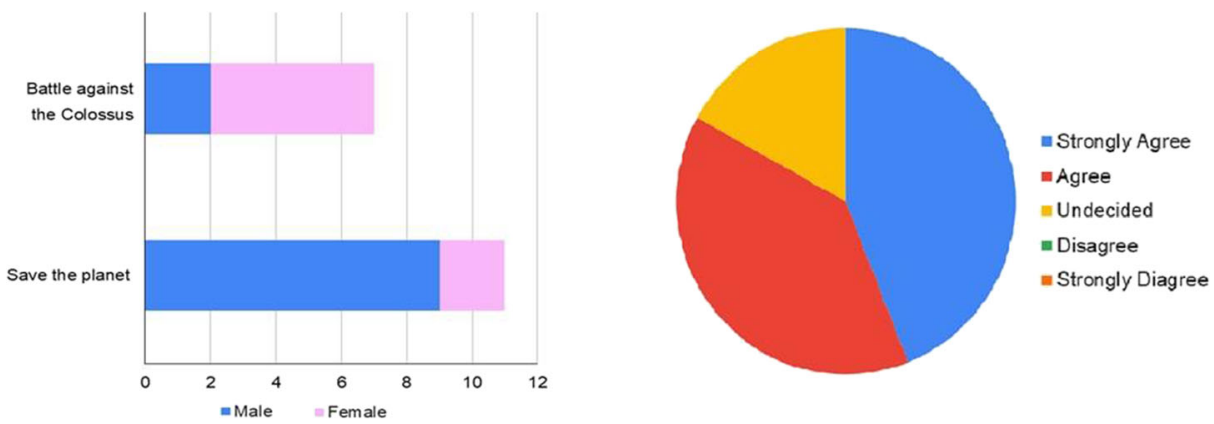

Fig. 11 (Left) Distribution of game choice between males and females. (Right) Using the smartphone was easy? 

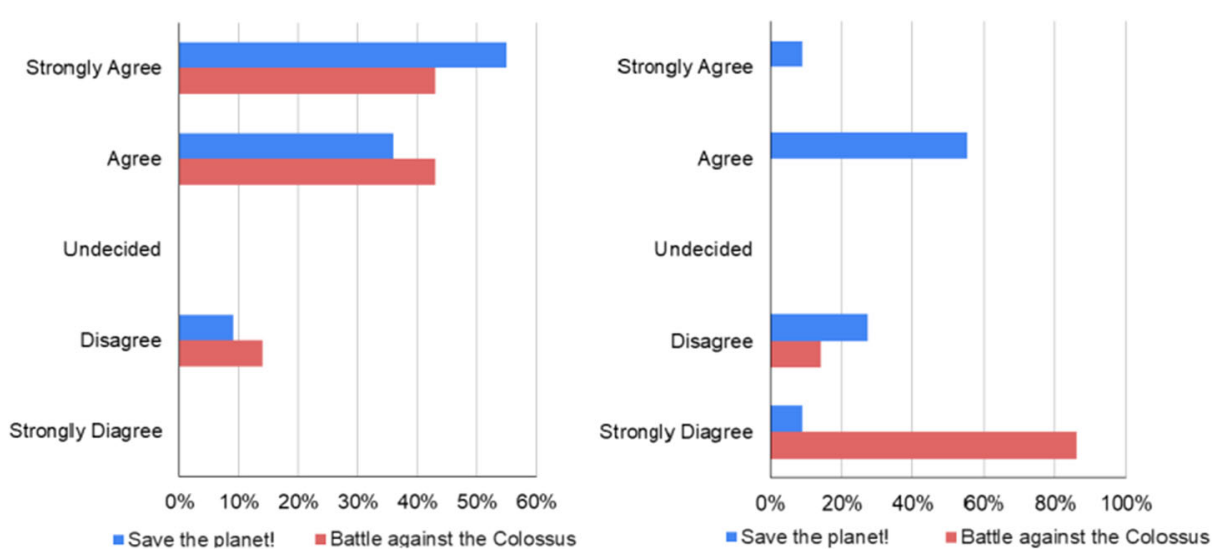

Fig. 12 (Left) I worked hard to get the exercises right. (Right) I got bored in the game

confirmed that the game pushed pupils to become faster in giving the answer, but having fun the kids didn't realize there was a countdown and they enjoyed the exercise. Another evidence of this result lied in the fact that pupils declared that they worked hard to get the exercise right (Fig. 12 left), in both games. Hence, the gameplay was not a distracting element, but on the contrary it gave pupils the chance to practice multiplication tables without caring about fatigue.

One inconsistent element that can be detected from the data is depicted in Figure (Fig. 12 right). Some children (64\%) using Save the planet! got bored during the game, but the $91 \%$ of the same children declare that was very funny repeating the multiplication tables with this game. Probably, the children were confused in giving the answer. The $94 \%$ of the whole sample stated that they would be happy to tell their friends about the game.

Concluding, the data revealed that children appreciated to practice with the multiplication tables using an augmented reality game, since it made amaz- ing the learning of multiplication tables that, as it is reported in literature, it is perceived by pupils as a repetitive and boring learning activity.

\section{Conclusions and future work}

Augmented Reality is one of the newest interaction modality adopted to en- hance learning experiences. The AR allows users to visualize virtual worlds combined with real environments. This is usually used to increase the engage- ment of the users to the applications, so they can feel that the digital objects are surrounding him/her. This article presents an application of AR technology as a support tool for learning multiplication tables. The possibility of mixing entertainment with AR techniques makes the tedious process of learning the multiplication tables more enjoyable and attractive for children, greatly im- proving their skills. The proposed serious game has been made with a Human Centered Design in order to develop an effective application both in terms of interaction and content, essential for educational purposes.

The game has been evaluated with children from 8-9 years old, a between subject-design was adopted to measure learning effectiveness and the users' engagement in the learning method. The results of data collected during the pilot test were very satisfactory, as expected the game allows the children to practice multiplication and have fun. Moreover, the 
measurement of learning gain was interesting since all pupils, both in the EG and in the CG, improved their knowledge in multiplication, confirming that after all, learning multipli- cation tables is only a matter of exercise and this game allows children to practice pleasantly.

From the usability point of view, the results were satisfactory. Most of the sample enjoyed the game and had no difficulty in using it. More in-depth evaluations will be necessary to understand why some students got bored with the game Save the planet!. Anyway, in the next future the AR interaction should be improved in order to be part of the game, not being only used for visualizing the game-play. This will make the game more appealing and exciting.

The results we present in this paper, correspond to those obtained in the pilot study we performed to measure the usability of the game. In the next future, we are planning to carry out an experimental study involving more participants to collect more data and perform more deep investigations.

Finally, other line to be carried out as future work deals with designing more social games, so children can interact with the game and also with other users, being able to perform some math competitions.

Acknowledgments This work was supported by the Spanish Ministry of Science and Technology (Project PID2019-106426RB-C32), the Universitat Jaume I research project (UJI-B2018-56), the UJI Educational Innovation Project 3805 GAMELAB and the Living Lab Planeta Debug supported by the Universitat Jaume I and the Carasso Foundation.

\section{References}

1. Almenara J, Osuna J, Llorente C, Martínez M (2019) Educational uses of augmented reality (ar): experiences in educational science. Sustainability 11(18)

2. Azuma R-T (1997) A survey of augmented reality. Presence: Teleoperators and Virtual Environments 6(4): 355-385

3. Cabiria J, Wankel C, Blessinger P (2012) Augmenting engagement: augmented reality in education. Emerald Group Publishing Limited 6:225-251

4. Cahyono B, Firdaus M-B, Budiman E, Wati M (2018) Augmented reality applied to geometry education. In Proc. 2nd East Indonesia Conf. Comput. Inf. Technol. (EIConCIT) pp. 299-303. Indonesia

5. Cerqueira JM, Sylla C, Moura JM, Ferreira L (2019) Learning basic mathematical functions with augmented reality. In book: Interactivity, Game Creation, Design, Learn- ing, and Innovation. pp. 508-513

6. Cerqueira JM, Moura JM, Martinho J, Sylla C, Ferreira LL (2020) An aug- mented reality mathematics serious game. In Proc. 1st International Computer Programming Education Conference (ICPEC). Portugal

7. CoolText Homepage, https://es.cooltext.com/. Last accessed 7 Jun 2020

8. Ergonomics of human-system interaction - Part 210: Human-centred design for interac- tive systems (ISO 9241-210:2019) (Endorsed by Asociación Española de Normalizacíon in November of 2019.)

9. Fambona J, Pascual M-Á, Madeira M-F (2012) Realidad aumentada una evoluci'on de las aplicaciones de los dispositivos m'oviles. Pixel-Bit Revista de Medios y Educación 41:197-210

10. Furió D, Juan M-C, Seguí I, Vivo R (2014) Mobile learning vs. traditional classroom lessons: A comparative study. Journal of Computer Assisted Learning 31:189-201

11. Ibáñez M-B, Portillo A-U, Cabada R-Z, Barrón M-L (2020) Impact of aug- mented reality technology on academic achievement and motivation of students from public and private Mexican schools. A case study in a middle-school geometry course. Comput Edu 145

12. Jones V, Jo J-H (2004) Ubiquitous learning environment: An adaptive teaching system using ubiquitous technology. Beyond the Comfort Zone: Proceedings of the 21st ASCILITE Conference. 468-474

13. Juan M-C, Furió D, Seguí I, Aiju N, Cano J (2011) Lessons learnt from an experience with an augmented reality iphone learning game. In Proceedings of the 8th Inter- national Conference on Advances in Computer Entertainment Technology. (52) ACM, Portugal 
14. Kang S, Shokeen E, Byrne V-L, Norooz L, Bonsignore E, Caro W-P, Froehlich J-E (2020) ARMath: augmenting everyday life with math learning. Proceedings of the $2020 \mathrm{CHI}$ Conference on Human Factors in Computing System, pp. 1-15. ACM, Honolulu

15. Keller J-M (2008) First principles of motivation to learn and e3-learning. Distance Education 29(2):175-185

16. Keller J-M (2016) Motivation, learning, and technology: applying the arcs-v motivation model. Participatory Educational Research (PER) 3(2):1-13

17. Liu, T-Y, Tan T.-H., Chu Y-L (2007) 2D barcode and augmented reality supported english learning system. In Proc. 6th IEEE/ACIS Int. Conf. Comput. Inf. Sci. (ICIS). pp. 5-10. IEEE, Melbourne

18. Mixamo Homepage, https://www.mixamo.com/. Last accessed 26 May 2020

19. PikPng Homepage, https://www.pikpng.com/. Last accessed 24 May 2020

20. Procedural Planets Unitylist, https://unitylist.com/p/lm7/procedural-planets. Last accessed 14 May 2020

21. Rossano, V., Roselli, T., Calvano, G. (2017, June). A serious game to promote en- vironmental attitude. In international conference on smart education and smart E- learning (pp. 48-55). Springer, Cham

22. Rossano V, Lanzilotti R, Cazzolla A, Roselli T (2020) Augmented reality to support geometry learning. IEEE Access 8:107772-107780

23. Schell J (2008) The art of game design: a book of lenses. Morgan Kaufmann Publishers Inc.

24. Sharples M, Corlett D, Westmancott O (2002) The design and implementation of a mobile learning resource. Personal and Ubiquitous Computing 6(3):220-234

25. Sketchfab Homepage, https://sketchfab.com/3d-models. Last accessed 4 Jun 2020

26. Tan K-T, Lewis E-M, Avis J-N, Withers J-P (2008) Using augmented reality to pro- mote an understanding of materials science to school children. In ACM SIGGRAPH ASIA 2008 educators programme. (2) ACM. Singapore

27. Tomaschko M, Hohenwarter M (2019) Augmented reality in mathematics education:The case of GeoGebra AR. In: Augmented reality in educational settings. 1st edition. pp. 325-346, Brill—Sense, The Netherlands

28. Unity Asset Store Homepage, https://assetstore.unity.com/. Last accessed 26 May 2020

29. Unity Shader Graph Homepage, https://unity.com/es/shader-graph. Last accessed 14 May 2020

30. Virvou M, Alepis E (2005) Mobile educational features in authoring tools for personalized tutoring. Computers Education 44:53-68

31. Vuforia Developer Library, https://ibrary.vuforia.com/content/vuforia-library/ en/articles/Training/groundplane-guide.Html. Last accessed 23 April 2020

32. Yusoff, Z, Dahlan, HM (2013). Mobile based learning: an integrated framework to sup-port learning engagement through Augmented Reality environment, International Confer- ence on Research and Innovation in Information Systems (ICRIIS), Kuala Lumpur, pp. 251-256

Publisher's note Springer Nature remains neutral with regard to jurisdictional claims in published maps and institutional affiliations. 